\title{
-NOTES-
}

\section{A UNIQUENESS THEOREM FOR THE DISPLACEMENT BOUNDARY-VALUE PROBLEM OF LINEAR ELASTODYNAMICS*}

By M. E. GURTIN (Brown University) AND R. A. TOUPIN (IBM T. J. Watson Research Center)

1. Introduction. The displacement equations of motion in the linear theory of homogeneous elastic solids, with reference to rectangular Cartesian coordinates and in the usual indicial notation**, are

where

$$
c_{i j k l} u_{k, l i}+f_{i}=\rho u_{i}^{(2)},
$$

$$
c_{i j k l}=c_{j i k l}=c_{k l i j} .
$$

The $u_{i}(\mathbf{x}, t)$ and $f_{i}(\mathbf{x}, t)$ are the Cartesian components of the displacement vector $\mathbf{u}(\mathbf{x}, t)$ and the body force $\mathbf{f}(\mathbf{x}, t) ; \mathbf{x}$ is a point of the closed region of space $R$ occupied by the body, and $t$ represents the time. The $c_{i j k l}$ are the components of the constant elasticity tensor $\mathbf{c}$ and $\rho$ denotes the constant mass density.

This note is concerned exclusively with the displacement boundary-value problem of elastodynamics. We therefore adjoin the boundary condition

$$
\mathbf{u}(\mathbf{x}, t)=\mathbf{u}^{*}(\mathbf{x}, t), \quad(\mathbf{x}, t) \mathbf{\varepsilon} B \times[0, \infty),
$$

as well as the initial conditions

$$
\mathbf{u}(\mathbf{x}, 0)=\mathbf{u}^{0}(\mathbf{x}), \quad \mathbf{u}^{(1)}(\mathbf{x}, 0)=\mathbf{v}^{0}(\mathbf{x}), \quad \mathbf{x} \varepsilon R .
$$

Here $B \times[0, \infty)$ is the Cartesian product of the boundary $B$ of $R$ and the time interval $[0, \infty)$, and $\mathbf{u}^{*}, \mathbf{u}^{0}$, and $\mathbf{v}^{0}$ are, respectively, the prescribed surface displacement, initial displacement, and initial velocity. We assume that $\mathbf{u}^{*}, \mathbf{u}^{0}, \mathbf{v}^{0}, \rho>0$, and $c_{i j k l}$ satisfying (1.2) are given. The displacement problem under consideration then consists in finding a twice continuously differentiable vector field $\mathfrak{u}$ on $R \times[0, \infty)$ which satsifies (1.1), (1.3), and (1.4).

The classical uniqueness theorem of Neumann [1] for the displacement problem rests on the assumption that the elasticity tensor is positive semi-definite, i.e., that

$$
c_{i j k l} e_{i j} e_{k l} \geq 0
$$

for every tensor $\dagger e_{i j}$. If the material is isotropic $\ddagger$ (1.5) is equivalent to the inequalities

$$
\mu \geq 0, \quad-1 \leq \sigma<\frac{1}{2}
$$

*Received March 25, 1964.

**Subscripts range over the integers $(1,2,3)$, summation over repeated subscripts is implied, and subscripts preceded by a comma indicate differentiation with respect to the corresponding space coordinate. Superscripts denote time differentiation. Letters in boldface designate tensors.

†In view of (1.2) we need not require e to be symmetric.

$\ddagger$ †.e.,

$$
c_{i j k l}=\mu\left[\delta_{i k} \delta_{j l}+\delta_{i l} \delta_{j k}+2 \sigma(1-2 \sigma)^{-1} \delta_{i j} \delta_{k l}\right],
$$

where $\delta_{i j}$ is the Kronerker delta. 
provided $\mu$ and $\sigma$ denote the shear modulus and Poisson's ratio of the material. Gurtin and Sternberg [2] have shown that for the isotropic case (1.6) may be relaxed without loss of uniqueness. Specifically they show that (1.6) may be replaced by the less stringent assumption

$$
\mu \geq 0, \quad-\infty<\sigma<\frac{1}{2}, \quad 1 \leq \sigma<\infty .
$$

Our objective here is to extend this result to the anisotropic case. In particular, we show that uniqueness of the displacement problem holds provided $\mathbf{c}$ is semi-stronglyeliptic, i.e.,

$$
c_{i j k l} \alpha_{i} \alpha_{k} \beta_{j} \beta l \geq 0
$$

for every pair of vectors $\alpha$, $\beta$. Under the assumption of isotropy (1.8) and (1.7) are equivalent. Moreover, for the general anisotropic case (1.8) is weaker than (1.5) since the latter condition implies the former, but not vice-versa.

Toupin and Bernstein [3] have shown that semi-strong-elipticity admits a simple intrinsic interpretation: an elastic body propagates plane waves with real speeds if and only if (1.8) holds.

2. The uniqueness theorem. In what follows we assume that $R$ is a bounded regular region of space, i.e., the boundary of $R$ consists of a finite number of non-intersecting closed regular surfaces, the latter term being used in the sense of Kellogg [4].

UnIQUENESS THEOREM. There is at most one solution to the displacement problem provided the elasticity tensor is semi-strongly elliptic.

Proof. In view of the linearity of (1.1), (1.3), and (1.4) it is sufficient to show that $\mathbf{f}=\mathbf{u}^{*}=\mathbf{u}^{0}=\mathbf{v}^{0}=\mathbf{0}$ implies $\mathbf{u}=\mathbf{0}$. Assume that the former holds. Recall the power identity

$$
\begin{gathered}
\int_{B} c_{i j k l} u_{k, l} n_{j} u_{i}^{(1)}(\mathbf{x}, t) d \mathbf{x}+\int_{R} f_{i} u_{i}^{(1)}(\mathbf{x}, t) d \mathbf{x}=U^{(1)}(t)+K^{(1)}(t) \quad(0 \leq t<\infty), \\
U(t)=\frac{1}{2} \int_{R} c_{i j k l} u_{i, j} u_{k, l}(x, t) d x \\
K(t)=\frac{1}{2} \int_{R} \rho u_{i}^{(1)} u_{i}^{(1)}(x, t) d x
\end{gathered}
$$

which may be verified using (1.1), (1.2), and the divergence theorem. This identity, together with $\mathbf{f}=\mathbf{u}^{*}=\mathbf{0}$ and the boundary condition (2.3), implies that $U^{(1)}(t)=$ $K^{(1)}(t)=0(0 \leq t<\infty)$. Next, since $\mathfrak{u}^{0}=\mathbf{v}^{0}=0$, the initial condition (2.4) yields $U(0)=K(0)=0$. Hence

$$
U(t)+K(t)=0 \quad(0 \leq t<\infty) .
$$

Suppose we could show that

$$
U(t) \geq 0 \quad(0 \leq t<\infty) .
$$

It would then follow from (2.4) that $K(t)=0(0 \leq t<\infty)$ which, because of (2.3), would imply that $\mathfrak{u}^{(1)}=0$ and hence that $\mathfrak{u}(\mathbf{x}, t)=\mathfrak{u}(\mathbf{x}, 0)=0$ for every $(\mathbf{x}, t) \varepsilon R \times[0, \infty)$. The proof therefore reduces to showing that the inequality (2.5) holds.

We establish (2.5) using a method due entirely to van Hove [5]. Extend the defini-

*We use $d \mathbf{x}$ to denote integration with respect to $\mathbf{x}$ in both surface and volume integrals. 
tion of $\mathfrak{u}$ to $E \times[0, \infty)$, where $E$ is the entire three-dimensional Euclidean space, by setting

$$
\mathbf{u}=\mathbf{0} \text { on }[E-R] \times[0, \infty)
$$

Then, by virtue of the assumed smoothness of $\mathfrak{u}$, it follows that, for each $t \geq 0, u_{i}(\mathbf{x}, t)$ and $u_{i, j}(\mathbf{x}, t)$ are both absolutely and square integrable over $E$ with respect to $\mathbf{x}$. Hence $u_{k}$ and $u_{k . \imath}$ possess, respectively, the three-dimensional Fourier transforms $\eta_{k}$ and $\eta_{k l}$ defined by

$$
\begin{aligned}
\eta_{k}(\mathbf{x}, t) & =(1 / 2 \pi)^{3 / 2} \int_{E} e^{i x_{i j} \nu_{j}} u_{k}(\mathrm{y}, t) d \mathbf{y}, \\
\eta_{k l}(\mathbf{x}, t) & =(1 / 2 \pi)^{3 / 2} \int_{E} e^{i x_{j} y_{j}} u_{k, l}(\mathrm{y}, t) d \mathbf{y} .
\end{aligned}
$$

Consequently, by (2.7), (2.8), the divergence theorem, and the fact that $\mathfrak{u}=0$ on $B \times[0, \infty)$, it follows that

$$
\eta_{k l}(\mathbf{x}, t)=-i x_{k} \eta_{l}(\mathbf{x}, t) .
$$

Next, a fundamental theorem of Fourier analysis (cf., e.g., Goldberg [5], Theorem 13E) yields

$$
\int_{E} u_{i, i}(\mathrm{x}, t) u_{k, l}(\mathrm{x}, t) d \mathrm{x}=\int_{E} \eta_{i j}^{c} \eta_{k l}(\mathrm{x}, t) d \mathbf{x}
$$

where the superscript $c$ denotes the complex conjugate. The functions $\eta_{k}$ are, in general, complex valued. Their real and imaginary parts will be denoted by $\eta_{k}^{\prime}$ and $\eta_{k}^{\prime \prime}$

$$
\eta_{k}=\eta_{k}^{\prime}+i \eta_{k}^{\prime \prime} \text {. }
$$

Then (2.6), (2.9), (2.10), and (2.11) imply

$$
\int_{R} u_{i, i}(\mathbf{x}, t) u_{k, l}(\mathbf{x}, t) d \mathbf{x}=\int_{E}\left[x_{i} x_{l}\left\{\eta_{i}^{\prime}(\mathbf{x}, t) \eta_{k}^{\prime}(\mathbf{x}, t)+\eta_{i}^{\prime \prime}(\mathbf{x}, t) \eta_{k}^{\prime \prime}(\mathbf{x}, t)\right\}\right] d \mathbf{x} .
$$

Now multiply (2.12) by $c_{i j k l}$ and use (1.8) to verify (2.5). This completes the proof.

\section{REFERENCES}

1. F. Neumann, Vorlesungen über die Theorie der Elasticität der festen Körper und des Lichtäthers, B. G. Teubner, Leipzig, 1885

2. M. E. Gurtin and Eli Sternberg, A note on uniqueness in classical elastodynamics, Quart. Appl. Math., 19 (1961) 169

3. R. A. Toupin and B. Bernstein, Sound waves in deformed perfectly elastic materials. Acousto-elastic effect., J. Acoust. Soc., 33 (1961) 216

4. O. D. Kellogg, Foundations of potential theory, J. Springer, Berlin, 1929

5. L. van Hove, Sur l'extension de la condition de Legendre du calcul de variations aux integrales multiples $d$ plusieurs fonctions inconnues, Konink. Nederl. Akad. Wetens., 50 (1947) 18

6. R. R. Goldberg, Fourier transforms, Cambridge University Press, London, 1961 